\title{
THEORY OF INFLOW CONTROL ON AN URBAN EXPRESSWAY SYSTEM
}

\author{
By Tsuna Sasaki* and Sho Myojin**
}

\section{INTRODUCTION}

The major interest in traffic control within urban area has been devoted to traffic regulation at at-grade intersection or in street system, and so few papers have been published on traffic control on an urban expressway system. The most parts of traffic regulations on street resort to signal control system, in which vehicle-actuated or co-ordinated signals are ordinarily used. Computer-controled area traffic control systems have been developed to cover whole urban area. In developing signal cotrol system, the major attention of traffic engineers has been usually focussed to signal operations through which they may realize the maximum or the minimum value of a given measure of effectiveness such as the total sum of stopped intervals, the total number of stoppings.

If traffic congestion is once caused on expressway without any control system, it will be forced to continue for a long period as compared with the case on street, since expressway is of access-controled system while street is not. Hence, ramp-control seems to be most important to control traffic flow on

Table 1 General remarks on traffic control on some expressways

\begin{tabular}{|c|c|c|c|c|}
\hline Country & City & $\begin{array}{l}\text { Name of road } \\
\text { (Control area) }\end{array}$ & $\begin{array}{c}\text { Surveying } \\
\text { system }\end{array}$ & Control items \\
\hline U.S.A. & Detroit & $\begin{array}{l}\text { John C. Rodge } \\
\text { Expressway }\end{array}$ & $\begin{array}{l}\text { ITTV. } \\
\text { vehicle } \\
\text { detector }\end{array}$ & $\begin{array}{l}\text { control for } \\
\text { lane, ramp and } \\
\text { speed limit }\end{array}$ \\
\hline$"$ & Houston & $\begin{array}{l}\text { Gulf Freeway } \\
(10.3 \mathrm{~km})\end{array}$ & $\begin{array}{l}\text { ITTV. } \\
\text { vehicle } \\
\text { detector }\end{array}$ & ramp-control \\
\hline$"$ & Chicago & $\begin{array}{l}\text { Eisenhower Ex- } \\
\text { pressway }(9.6 \mathrm{~km} \\
\text { in a single direct- } \\
\text { ion) }\end{array}$ & $\begin{array}{l}\text { vehicle } \\
\text { detector }\end{array}$ & ramp-control \\
\hline$"$ & Seatle & $\begin{array}{l}\text { Seatle Freeway } \\
(12.0 \mathrm{~km})\end{array}$ & ITTV. & $\begin{array}{l}\text { control for } \\
\text { lane and ramp }\end{array}$ \\
\hline England & London & $\begin{array}{l}\text { Mortorway M } 4 \\
(3.2 \mathrm{~km})\end{array}$ & $\begin{array}{l}\text { vehicle } \\
\text { detector }\end{array}$ & $\begin{array}{l}\text { instruction } \\
\text { for detour }\end{array}$ \\
\hline $\begin{array}{c}\text { West } \\
\text { Germany }\end{array}$ & München & $\begin{array}{l}\text { München Saltburg } \\
\text { Autobahn ( } 30 \mathrm{~km} \\
\text { to the south of } \\
\text { München) }\end{array}$ & ITTV. & $\begin{array}{l}\text { speed control } \\
\text { instruction } \\
\text { for detour }\end{array}$ \\
\hline Japan & & $\begin{array}{l}\text { Meishin Express- } \\
\text { way }(180 \mathrm{~km})\end{array}$ & & speed control \\
\hline$"$ & Tokyo & $\begin{array}{l}\text { Miyake-saka tun- } \\
\text { nel on Metropoli- } \\
\text { tan Expressway }\end{array}$ & ITTV. & $\begin{array}{l}\text { instruction } \\
\text { for detour }\end{array}$ \\
\hline
\end{tabular}

* Department of Transportation Engineering, Faculty of Engineering, Kyoto University.

** Department of Transportation Engineering, Faculty of Engineering, Kyoto University. expressway.

Table 1 shows some of the traffic control systems on experssway that have ever been developed in some countries. From Table 1 we see that controls on expressway are conducted mainly for lanes, ramps and speed limits. From the theoretical point of view inflow control (ramp-control) seems to be most interesting of the preceding controls. In this paper inflow control system will be concerned.

\section{OBJECTIVES OF CONTROL AND CONTROLLABLE QUANTITIES}

An efficient control should not be achieved without examinations of what is aimed at, what are controllable and what are the available informations. We settle our objectives of control of traffic demand on expressway as follows :

1) The maximum allowable inflow is to be reached under the constraint that the realizcd trafic flow should not be in excess of the traffic capacity over every section. If urban expressway is under the application of a uniform toll-rate system, as is generally the case with urban expressway, the maximum inflow means the maximum gross revenue.

2) The dead traffic flow caused by any accident should be revived as soon as possible. The emergency countermeasures for accident will include controls for ramps, traffic lanes, route choices and speed limits.

For those objectives of control, we have the following controllable quantities :

1) inflow through each-ramp,

2) speed limit over every section, and

3) traffic lanes or route choices.

In preparation for control, observations should be made of the items :

1) inflow through every on-ramp,

2) outflow through every off-ramp,

3) traffic flow over every section,

4) travelling speed over every section and

5) traffic density (or occupancy) over every section.

We may have inflow control so that we should attain our objectives by use of those observed informations. 


\section{MATHEMATICAL DESCRIPTION OF TRAFFIC FLOW ON EXPRESSWAY}

Our objectives of control could not be achieved without any specified description of traffic flow on expressway. In the following, we are concerned with different descriptions.

\section{(1) Branching Probability Matrix Method}

This is the case with an absorbing Markov chain, which we have ever applied to the description of trip distribution. ${ }^{1)}$ In the following will be investigated two states of traffic flow; steady state and unsteady state.

We devide an expressway system into $m$ sections in a single direction, numbering them $1,2, \cdots, m$. Let $q_{i j}$ denote branching probability of traffic flow from section $i$ to section $j$, and $x_{i}(i=1,2, \cdots, m)$ be traffic volume over section $i$. We denote traffic volume over expressway by a row vector

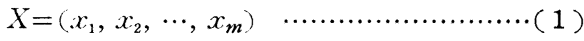

and denote inflows and outflows by row vectors, respectively,

$$
\left.\begin{array}{l}
u=\left(U_{1}, U_{2}, \cdots, U_{k}\right) \\
v=\left(V_{1}, V_{2}, \cdots, V_{r}\right)
\end{array}\right\}
$$

where,

$U_{i}=$ inflow through $\operatorname{ramp} i(i=1,2, \cdots, k)$

$V_{j}=$ outflow through $\operatorname{ramp} j(j=1,2, \cdots, r)$

$k=$ the number of on-ramps

and

$r=$ the number of off-ramps

Our problem is to express traffic flow over each section under a trip distribution which will be estimated in paragraph 4 . If we regard on-ramps and off-ramps as sources and sinks respectively as is popular in absorbing Markov chain, we may apply the absorbing Markov chain to the problem. The branching probability matrix $P$ may be written in canonical form of

$$
P=\left(\begin{array}{lll}
I & 0 & 0 \\
R_{1} & 0 & Q_{1} \\
R_{2} & 0 & Q_{2}
\end{array}\right)
$$

where,

$R_{1}=\left(r_{i j^{(1)}}\right), r_{i j}{ }^{(1)}=$ the probability of an inflow from on-ramp $i$ to off-ramp $j, \quad(i=1,2$, $\cdots, k, j=1,2, \cdots, r)$

$R_{2}=\left(r_{i j}{ }^{(2)}\right), r_{i j}{ }^{(2)}=$ the branching probability of traffic flow from section $i$ to off-ramp $j,(i=1,2, \cdots, m, j=1,2, \cdots, r)$

$Q_{1}=\left(q_{i j}{ }^{(1)}\right), q_{i j^{(1)}}=$ the probability of an inflow from on-ramp $i$ to section $j,(i=1,2, \cdots$, $k, j=1,2, \cdots, m)$

$Q_{2}=\left(q_{i j}{ }^{(2)}\right), q_{i j}{ }^{(2)}=$ the branching probability of traffic flow from section $i$ to section $j$, $(i=1,2, \cdots, m, j=1,2, \cdots, m)$

and

$I=(r \times r)$ unit matrix

In matrix $P$, off-ramp, on-ramp and section are put in turn both in row and in coluumn. With those notations, traffic flows over sections are given by

$$
X=u Q_{1}\left(I-Q_{2}\right)^{-1} \ldots \ldots \ldots \ldots \ldots \ldots \ldots \ldots \ldots \ldots(4)
$$

and outflows through ramps are given by

$$
v=u\left[R_{1}+Q_{1}\left(I-O_{2}\right)^{-1} R_{2}\right] \cdots \cdots \cdots \cdots \cdots \cdots \cdots \cdots(5)
$$

both in steady state ${ }^{2)}$.

The branching probability may be estimated through observation, though it is usually expensive. However, if trip distributions between ramps are given someway, we may calculate the branching probability by assignning the trips between ramps to the expressway network (the shortest route method may be of practical use in assignning). Estimation model for trip distributions will be shown later in paragraph 4. The theoretical control seems to be impossible without assuming that the estimated branching probability should be fixed during a specified interval (about an hour or so). Here we assume the branching probability being fixed. Our objective of control is to find the maximum inflow under constraint that the assigned traffic flow should never be in excess of the capacity over every section. If we put

$$
C=\left(C_{1}, C_{2}, \cdots, C_{m}\right)
$$

where,

$$
C_{i}=\text { traffic capacity over section } i, \quad(i=1,2,
$$$$
\cdots, m)
$$

then our problem is to find $U_{i}^{\prime} s$ such that realize

$$
\max \left(U_{1}+U_{2}+\cdots+U_{k}\right)
$$

subject to

$$
u Q_{1}\left(I-Q_{2}\right)^{-1} \leqq C^{\prime}
$$

Hence we can obtain the allowable inflows through ramps by use of Linear Programming. It is noted that the branching probability matrices, $\boldsymbol{Q}_{1}$ and $\boldsymbol{Q}_{2}$, are assumed to be fixed during a certain interval of time. It is the fact that the branching probability matrices, $Q_{1}$ and $Q_{2}$, should not always be fixed, and that the real branching probability would never be known so far as the probability is calculated according to the estimated trip distributions.

Let row vector $Y$ be the real traffic flow observed at a certain time, of which element is the actual traffic over a section. We may have

$$
Y=X+\varepsilon
$$

where $\varepsilon$ is the error to be introduced for eliminating the difference between $X$ and $Y$. Since $Y$ is timevariant even during a fixed interval though $X$ is fixed during the interval, the error vector $\varepsilon$ is timevariant. Using the error vector $\boldsymbol{\varepsilon}$, our problem may be reduced to finding $U_{i}{ }^{\prime} s(i=1,2, \cdots, k)$ such that realize

$\max \left(\Sigma U_{i}\right)$

subject to

$$
u Q_{1}\left(I-Q_{2}\right)^{-1}+\varepsilon \leqq C
$$

Let's call such inflow control the Linear Programming method. 
So far we examined an inflow control for steady flow. It is the fact that the actual flow may not always be considered steady. Traffic flow over a section is composed of inflows which apper there with various time lags that will be caused by travelling times necessary from on-ramps to the section. If the inflows are time-variant, so is traffic flow over the section. Considering the time lags, we attempt to describe traffic flow over expressway network in the following.

Suppose that the expressway in question consists of $m$ sections which require a constant time to pass through. As a unit of time we take an interval during which the section is passed through.

If we denote traffic flow over section $i$ and inflow through ramp $k$ at time $t$ by $x_{i}(t)$ and $U_{k}(t)$ respectively, we have

$$
\begin{aligned}
x_{i}(t)= & \sum_{k} U_{k}(t-1) q_{k i}{ }^{(1)}+\sum_{j} x_{j}(t-1) q_{j i}{ }^{(2)}, \\
& (i=1,2, \cdots, m)
\end{aligned}
$$

where $q_{k i}{ }^{(1)}$ and $q_{j i}{ }^{(2)}$ are the entries of the branching probability matrices $Q_{1}$ and $Q_{2}$, respectively. With matrix notation we have

$$
X(t)=u(t-1) Q_{1}+X(t-1) Q_{2}
$$

and similarly we have

$$
X(t-\tau+1)=u(t-\tau) Q_{1}+X(t-\tau) Q_{2}
$$

which, multiplied both sides by $Q_{2}^{-},{ }^{(t-\tau+1)}$ is reduced to

$$
\begin{aligned}
& X(t-\tau+1) Q_{2}{ }^{-(t-\tau+1)}-X(t-\tau) Q_{2}{ }^{-(t-\tau)} \\
& =u(t-\tau) Q_{1} Q_{2}^{-(t-\tau+1)} \ldots \\
& \text { If we put } \\
& X(t-\tau) Q_{2}{ }^{-(t-\tau)} \equiv H(t-\tau)
\end{aligned}
$$

it follows

$$
H(t-\tau+1)-H(t-\tau)=u(t-\tau) Q_{1} Q_{2}{ }^{-(t-\tau+1)}
$$

This is the difference equation of the first order and the solution of which is given by

$$
H(t)-H(t-\tau)=\sum_{l=1}^{\tau} u(t-l) Q_{1} Q_{2}{ }^{-(t-l+1)} \cdot \cdot(12)
$$

Multiplying both sides of Eq. (12) by $Q_{2}{ }^{t}$, we get

$$
X(t)-X(t-\tau) Q_{2}{ }^{\tau}=\sum_{l=1}^{\tau} u(t-l) Q_{1} Q_{2}{ }^{l-1}
$$

Finally we have

$$
X(t)=\sum_{l=1}^{\tau} u(t-l) Q_{1}{Q_{2}}^{l-1}+X(t-\tau) Q_{2}{ }^{\tau} \cdots
$$

Eq. (13) is corresponding to Eq. (4) that describes traffic flow in steady state. If inflows are steady, that is, if

$$
u(t-1)=u(t-2)=\cdots=u(t-\cdots \tau)=u
$$

then Eq. (13) is reduced to Eq. (4), because the first and the secod terms on the right side of Eq. (13) converge to $u Q_{1}\left(I-Q_{2}\right)^{-1}$ and 0 respectively as $\tau$ approaches to infinity. Note that Linear Programming control is hardly to apply to unsteady flow since the objective function may not be settled successfully.

\section{(2) Route Matrix Method}

The branching probabability matrix method is available only for the trip distribution in steady state. However, the assumption that the branching proba- bility from a certain section to any one of the successives is in the steady state may not be valid for the real traffic flow, because the probability should vary according to the variation in the origin-destination composition of the flow over the section and such variation is inevitable in the actual flow. So the inflow control in use of the branching probability matrix includes possibility of failure in getting what we have expected to realize. However, it seems that there may be still a hope of estimating successfully the branching probabilities following after the origindestination composition of trips.

Let's describe traffic flow on expressway in another way. In the following we investigate the route matrix method instead of the branching probability matrix method. In the route matrix method, estimation of trip distributions between ramps is indispensable.

We assume that trip distributions between ramps are given. Suppose there has been an inflow of a single vehicle through ramp $i$. Obviously there are $r$ probable destinations (off-ramps) of the vehicle to resach. A route matrix is obtained for on-ramp $i$ such that

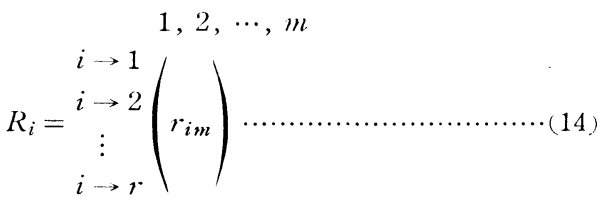

The route matrix $R_{i}$ is $(r \times m)$ matrix, in which im-entry $r_{i m}$ is equal to 1 if and only if the route from on-ramp $i$ to off-ramp $j(j=1,2, \cdots, r)$ includes section $m(m=1,2, \cdots, m)$, and is equal to 0 otherwise. Of course, in matrix $R_{i}$ origin-destinations $i \rightarrow j^{\prime} s$ are placed in row and sections $j^{\prime} s$ are in column. We have $k$ kinds of route matrices because there are $k$ number of on-ramps. We denote those matrices by $R_{1}, R_{2}, \cdots, R_{k}$.

If we denote trip distributions of inflow through ramp $i$ by a row vector

$$
p_{i}=\left(p_{i_{1}}, p_{i_{2}}, \cdots, p_{i_{r}}\right)
$$

where,

$$
\begin{aligned}
p_{i j}= & \text { transition probability of a trip from on- } \\
& \text { ramp } i \text { to off-ramp } j,(j=1,2, \cdots, r)
\end{aligned}
$$$$
\sum_{j}^{\prime} p_{i j}=1 \text {, }
$$

then we have

$$
p_{i} R_{i}
$$

which implies the expected flow to appear over expressway network when a single vehicle has inflowed through $\operatorname{ramp} i$. Since there are $k$ kinds of route matrices, we have

$$
Q=\left(\begin{array}{c}
p_{1} R_{1} \\
p_{2} R_{2} \\
\vdots \\
p_{k} R_{k}
\end{array}\right)=\left(Q_{i j}\right),(k \times m)
$$

which shows the overall expected traffic flow over ex- 
pressway network when a single vehicle has inflowed through each ramp. Hence, if we use row vector $u$ as the notation of the actual inflows, we have for the gross actual traffic flow

$$
X=u Q
$$

Similarly to the foregoing, our problem has been reduced to determining $U_{i}{ }^{\prime} s$ so as to maximize $\Sigma U_{i}$ under the constraint of

$$
u Q+\varepsilon \leqq C
$$

which is nothing but Linear Programming control.

Let's call the matrix $Q$ given by (16) the unit inflow matrix in its own sense. The unit inflow matrix may be quite insensitive to the variation in the trip distributions of the actual inflows. The inflow vector $u$ is variable according to the variation in inflows. Therefore the traffic flow given by Eq. (17) is of sufficient reliability. Remind that the control in use of branching probability matrix is insecure because the branching probabilities are hardly to follow after the variation in the trip distributions between ramps, and we see that the control in use of route matrix (inequality (18)) is the most improved control method among what we have reached.

In the foregoing we investigated on the control for steady flow, now we attempt to describe the constraint for control for unsteady flow. By $\tau_{i j}$ denote the travelling time from on-ramp $i$ to a fixed section $j$, and traffic flow over section $j$ at a certain time $t$ is given by

$$
x_{j}(t)=\sum_{i=1}^{k} U_{i}\left(\ell-\tau_{i j}\right) Q_{i j}, \quad(j=1,2, \cdots, m)
$$

where $Q_{i j}$ is $i j$-entry of the unit inflow matrix. In use of Eq. (19) the constraint for coctrol for unsteady flow is stated as

$$
\sum_{i=1}^{k} U_{i}\left(t-\tau_{i j}\right) Q_{i j}+\varepsilon_{j} \leqq C_{j}, \quad(j=1,2, \cdots, m)
$$

which shows that traffic flow should never be in excess of the capacity over every section. The matrix notation of inequality (20) is just complicated. The objective function is hardly to settle successfully.

\section{ESTIMATION OF TRIP DISTRIBUTI- ONS BETWEEN RAMPS}

In the last paragraph our investigation has not gone into estimation of trip distributions between ramps. For our inflow control the estimation is indispensable. The estimation should not be required if we could interview every driver at tollgates to ask which exit-ramp he is going to choose, because we might control inflows using the informations obtained by the interview. It is, however, supposed that such an interview is really troublesome. So we have to estimate trip distributions between ramps someways.

Investigation has been made as to good use of in- flows and outflows through ramps. At present it seems impossible to estimate the origin-destination composition of inflows during a very short interval of time, since we have no informations available to estimate the one fluctuating continually. So we should treat of gross flows that is presumably in steady state. The following investigation in trip distributions will be concerned with such gross inflows and outflows through ramps during a suitably long interval of time. A good fit of the estimation will be shown later to the actual trips. We apply the entropy maximization method $^{3}$ to the estimation of trip distributions.

By $x_{i j}$ we denote the number of trips from onramp $i$ to off-ramp $j$. Our problem is to estimate $x_{i j}$ by use of both inflow vector $u$ and outflow vector $v$ given.

Origin-destination table reminds us that we may write

$$
\left.\begin{array}{l}
\sum_{j=1}^{r} x_{i j}=U_{i}, \quad(i=1,2, \cdots, k) \\
\sum_{i=1}^{k} x_{i j}=V_{j}, \quad(j=1,2, \cdots, r)
\end{array}\right\}
$$

If we put

$$
\sum_{i=1}^{k} U_{i}=\sum_{j=1}^{r} V_{j}=T
$$

we have the following relations

$$
\begin{aligned}
& x_{i j}=U_{i} p_{i j}=T u_{i} p_{i j}, \quad\left(\Sigma u_{i}=1\right) \\
& V_{j}=T v_{j}, \quad\left(\Sigma v_{j}=1\right)
\end{aligned}
$$

where,

$$
p_{i j}=\text { probability of occurrence of a trip }
$$$$
\text { from ramp } i \text { to ramp } j \text {. }
$$

Using the relations of Eqs. (21), we have

$$
\left.\begin{array}{l}
\sum_{j=1}^{r} p_{i j}=1, \quad(i=1,2, \cdots, k) \\
\sum_{i=1}^{k} u_{i} p_{i j}=v_{j}, \quad(j=1,2, \cdots, r)
\end{array}\right\}
$$

The distribution of trip length on an urban expressway seems to have a peak on a certain trip length, showing a monotonous decrease around this peak length. So we assume an apriori probability of occurrence of a trip of length $t_{i j}$ (in minute) as follows :

$$
p_{i j}{ }^{\prime}=\alpha u_{i} v_{j}\left(t_{i j}\right)^{\beta} e^{-\gamma t_{i j}}
$$

where,

$$
\begin{aligned}
& u_{i}=\text { normalized inflow through on-ramp } i \\
& v_{j}=\text { normalized outflow throuh off-ramp } j \\
& t_{i j}=\text { travel time (trip length on expressway) }
\end{aligned}
$$$$
\text { from } \operatorname{ramp} i \text { to ramp } j \text { (in minute) }
$$

and $\alpha, \beta$ and $\gamma$ are coefficients. Notice that we have assumed an exponential from instead of a gravity from. A joint probability of occurrence of trips between ramps should be given by

$$
p=\frac{T !}{\Pi\left(x_{i j} !\right)} \Pi\left(p_{i j}\right)^{x_{i j}}
$$

Our problem is to determine $x_{i j}{ }^{\prime} s$ so as to maximize the joint probability $p$, since such a pattern of 
OD table is most probable under the given marginal distribution (Notice that the marginal distribution is given as an inflow vector $u$ and an outflow vector $v)$. To maximize $p$ is equivalent to maximizing $\log (p)$. Finally, using Stiring Formula, to maximize $\log (p)$ is proved equivalent to determining $p_{i j}{ }^{\prime} s$ so as to maximize

$$
H+\beta \overline{\log t}-\gamma \bar{t}
$$

Table 2 Observed trip distribution (Hanshin Expressway)

\begin{tabular}{c|r|r|r|r|r|r|r|r|r|}
\hline off-ramp & $1^{\prime}$ & $2^{\prime}$ & $3^{\prime}$ & $4^{\prime}$ & $5^{\prime}$ & $6^{\prime}$ & $7^{\prime}$ & Total \\
\hline on-ramp & & 33 & 345 & 848 & 1142 & 885 & 24 & 65 & 3342 \\
\hline 1 & 11 & 101 & 293 & 412 & 228 & 10 & 24 & 1709 \\
2 & 10 & 18 & 71 & 165 & 265 & 19 & 19 & 587 \\
3 & 4 & 4 & 7 & 25 & 42 & 7 & 55 & 144 \\
4 & 265 & 88 & 13 & 39 & 40 & 267 & 1735 & 2447 \\
5 & 328 & 114 & 14 & 25 & 40 & 168 & 1769 & 2458 \\
6 & 62 & 32 & 17 & 12 & 8 & 42 & 475 & 648 \\
7 & 713 & 702 & 1263 & 1820 & 1508 & 537 & 4162 & 10705 \\
\hline Total & 712 & & & &
\end{tabular}

The name of on-ramp

1 =Umeda $\quad 2$ =Dozima $\quad 3=$ Koraibash $\quad 4=$ Nagahor

$5=$ Ebisu-cho $\quad 6=$ Minato-machi $\quad 7=$ Yotsubashi

(vehicles/day), surveyed on April, 18 th 1967.

Table 3 Estimated trip distribution with exponential from (Hanshin Expressway)

\begin{tabular}{c|r|r|r|r|r|r|r|r}
\hline $\begin{array}{c}\text { off-ramp } \\
\text { on-ramp }\end{array}$ & $1^{\prime}$ & $2^{\prime}$ & $3^{\prime}$ & $4^{\prime}$ & $5^{\prime}$ & $6^{\prime}$ & $7^{\prime}$ & Total \\
\hline 1 & 330 & 401 & 711 & 969 & 724 & 37 & 171 & 3343 \\
2 & 24 & 73 & 225 & 353 & 300 & 18 & 86 & 1079 \\
3 & 8 & 4 & 72 & 177 & 212 & 19 & 95 & 587 \\
4 & 3 & 2 & 6 & 31 & 57 & 7 & 38 & 144 \\
5 & 150 & 84 & 72 & 77 & 75 & 246 & 1742 & 2446 \\
6 & 156 & 106 & 131 & 155 & 101 & 176 & 1634 & 2459 \\
7 & 41 & 33 & 46 & 58 & 40 & 33 & 396 & 647 \\
\hline Total & 712 & 703 & 1263 & 1820 & 1509 & 536 & 4162 & 10705 \\
\hline
\end{tabular}

The name of off-ramp

$1^{\prime}=$ Kitahama $\quad 2^{\prime}=$ Hommachi $\quad 3^{\prime}=$ Dotonbori

$4^{\prime}=$ Yuhigaoka $\quad 5^{\prime}=$ Namba $\quad 6^{\prime}=$ Tosabori $\quad 7^{\prime}=$ Deiribashi

(vehicles/day), $\beta=3.20456, \gamma=0.73792$, (Eq. (23))

Table 4 Travel time between ramps (Hanshin Expressway) (minute)

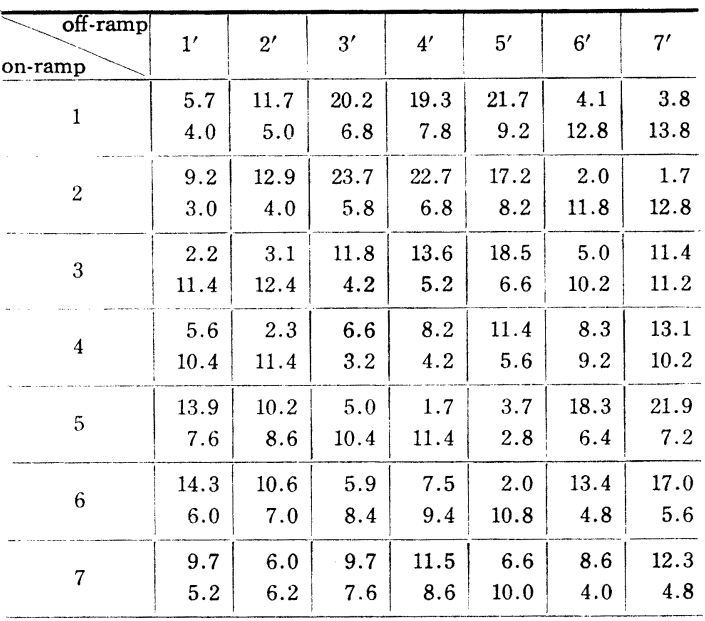

the upper =alternative street $\left(t_{i j}{ }^{\circ}\right)$

the lower $=$ expressway $\left(t_{i j}\right)$ where,

$$
\left.\begin{array}{l}
H=-\Sigma \Sigma u_{i} p_{i j} \log \left(p_{i j}\right) \\
\overline{\log t}=\Sigma \Sigma u_{i} p_{i j} \log \left(t_{i j}\right) \\
\bar{t}=\Sigma \Sigma u_{i} p_{i j} t_{i j}
\end{array}\right\}
$$

In Eqs. (25), $H$ is so called entropy in information theory. The number $x_{i j}$ of trips from ramp $i$ to $\operatorname{ramp} j$ is given by $T u_{i} p_{i j}$.

Table 2 shows the actual trip distribution between ramps on Hanshin Expressway which is surveyed on April 18 th, 1967, when the expressway system was opened only by a single circuit as shown in Fig. 1.

The estimated trip distribution is shown in Table 3 , which shows a good fit to the actual one.

Travelling time (trip length in minute) between ramps on expressway is given in Table 4 . Table 4. also includes time $t_{i j}{ }^{\circ}$ which is required to travel along alternative street. The travelling time $t_{i j}{ }^{\circ}$ should better be taken into consideration in order to improve the estimation, since the alternative street is considered sure to give any influence on trip distribution on expressway.

Using $t_{i j}{ }^{\circ}$, we assume an apriori probability of occurrence of a trip between $\operatorname{ramp} i$ and $\operatorname{ramp} j$ as follsws :

$$
p_{i j}{ }^{\prime}=\alpha u_{i} v_{j}\left(t_{i j}\right)^{\beta} e^{-\gamma t_{i j}}\left(t_{i j}{ }^{\circ} / t_{i j}\right)^{\delta} \cdots \cdots \cdots \cdots(26)
$$

in which $\alpha, \beta, \gamma$ and $\delta$ are coefficients. The objective function is given by

$$
H+\beta \overline{\log t}-\gamma \bar{t}+\delta \overline{\log \left(t^{\circ} / t\right)}
$$

where,

$$
\overline{\log \left(t^{\circ} / t\right)}=\Sigma \nu^{\prime} u_{i} p_{i j} \log \left(t_{i j}{ }^{\circ} / t_{i j}\right)
$$

Table 5 is the result obtained in this way, which, as compared with Table 3 , shows an improved fit to the actual distribution. The parameters finally decided are

$$
\alpha=0.01009, \beta=4.20368, \gamma=0.57162
$$

and

$$
\delta=0.83848
$$

Further investigation in trip distribution may be of worth in order to reach a better estimation. With a view to controlling, however, it is also worthy

\begin{tabular}{|c|c|c|c|c|c|c|c|c|}
\hline $\begin{array}{l}\text { off-ramp } \\
\text { on-ramp }\end{array}$ & $1^{\prime}$ & $2^{\prime}$ & $3^{\prime}$ & $4^{\prime}$ & $5^{\prime}$ & $6^{\prime}$ & $7^{\prime}$ & Total \\
\hline 1 & 178 & 329 & 725 & 1004 & 884 & 40 & 182 & 3342 \\
\hline 2 & 52 & 88 & 254 & 379 & 259 & 9 & 37 & 1078 \\
\hline 3 & 6 & 5 & 66 & 140 & 185 & 16 & 166 & 584 \\
\hline 4 & 5 & 1 & 7 & 21 & 34 & 9 & 65 & 142 \\
\hline 5 & 191 & 106 & 55 & 28 & 65 & 243 & 1762 & 2450 \\
\hline 6. & 230 & 143 & 101 & 163 & 40 & 185 & 1599 & 2461 \\
\hline 7 & 50 & 29 & 54 & 85 & 41 & 34 & 355 & 648 \\
\hline Total & 712 & 701 & 1262 & 1820 & 1508 & 536 & 4166 & 10705 \\
\hline
\end{tabular}
of investigating in traffic flow over expressway so as to be of good fitness to the actual flow. Fig. 1 shows the surveyed flow over the expressway. Fig. 2 and Fig. 3 are obtained by assingnning OD trips

Table 5 Estimated trip distribution with travel time ratio (Hanshin Expressway) 


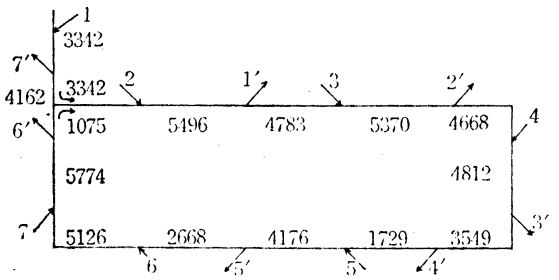

Fig. 1 Traffic flow (observed), (vehicles/day)

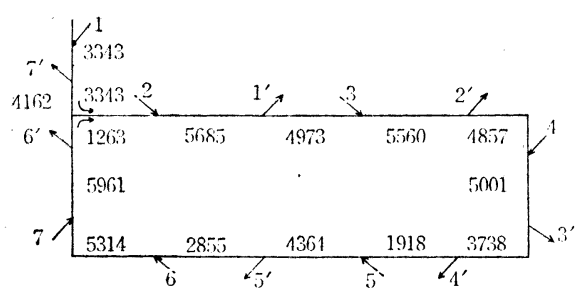

Fig. 2 Traffic flow (estimated with exponential form), (vehicles/day)

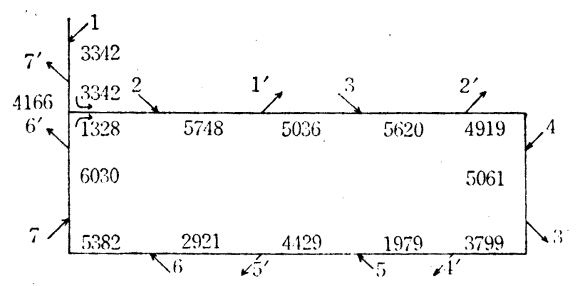

Fig. 3 Traffic flow (estimated with travee time ratio), (vehicles/day)

shown in Table 3 and Table 5 respectively. It is noticeable that traffic flows shown in Fig. 2 and Fig. 3 are both overestimated over every section by a constant value; about 190 and 250 number of vehicles respectively. It suggests us that we may have just the same flow as the actual one by shifting the value of any of parameters that are involved in the assumed form of apriori probability. Suppose that we shift one or more of these values, and trip distribution between ramps will vary accordingly. For instance, if we shift the value of the parameter $r$ involved in the assumed form of (26) toward a larger value keeping the others constant, we will have a shorter trip length on which trip distribution has its peak. That is to say, by shifting the value of the parameter $r$ toward the larger one, the trip of shorter length increases and the one of longer length decreases each in the number. Remind that the expressway under investigation is open only by a single circuit and that we have fixed the inflow vector $u$ and the outflow vector $v$ (marginal distribution of OD table), then we may easily perceivi that it is possible to realize just the same flow as the actual one by such a shifting.

Keeping the values of coefficients $\beta$ and $\delta$ constant, we shift the value of coefficient $r$ alone in Eq. (26) (in which travelling time ratio is included) to find the probable value. Finally we have the value
Table 6 Estimated trip distribution with traffic flow fitted to the observed one (Hanshin Expressway)

\begin{tabular}{c|r|r|r|r|r|r|r|r}
\hline $\begin{array}{c}\text { off-ramp } \\
\text { on-ramp }\end{array}$ & $1^{\prime}$ & $2^{\prime}$ & $3^{\prime}$ & $4^{\prime}$ & $5^{\prime}$ & $6^{\prime}$ & $7^{\prime}$ & Total \\
\hline 1 & 224 & 376 & 751 & 1026 & 842 & 23 & 100 & 3342 \\
2 & 66 & 100 & 260 & 384 & 244 & 5 & 20 & 1079 \\
3 & 4 & 3 & 82 & 170 & 209 & 11 & 108 & 587 \\
4 & 3 & 1 & 11 & 30 & 44 & 7 & 49 & 145 \\
5 & 162 & 82 & 38 & 19 & 112 & 251 & 1782 & 2446 \\
6 & 206 & 117 & 77 & 124 & 28 & 202 & 1704 & 2458 \\
7 & 47 & 25 & 43 & 67 & 30 & 39 & 397 & 648 \\
\hline Total & 712 & 704 & 1262 & 1820 & 1509 & 538 & 4160 & 10705 \\
\hline$\beta=4.20368$, & $r=0.67000, \delta=0.83848$, (Eq. (26)), (vehicles/day)
\end{tabular}

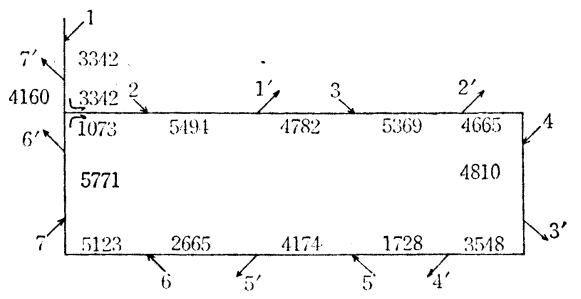

Fig. 4 Traffic flow (estimated with traffic flow fitted to the observed one), (vehicles/day) $r=0.670$

Then we obtained OD table and traffic flow shown in Table 6 and in Fig. 4 respectively. The traffic flow in Fig. 4 is in almost full coincidence with that in Fig. 1. Trip distribution estimated here seems to have been improved to considerable extent except for a few cells. It suggests us that we may estimate trip distribution keeping the calculated traffic flow corresponding with the actual one.

Our method of estimating OD trip on expressway requires as information both of the surveyed traffic flows and the surveyed in- and outflows. In detail, at first the values of coefficients in objective function are decided so that the calculated traffic flow should be fitted to the surveyed one as well as possible, and next estimation of OD trip may be made by use of those values and the surveyed in- and outflows. From the practical point of view our method is satisfactorily effective.

We have reached the estimation of trip distribution that realizes almost the same flow as the actual one over every section. Fig. 5 shows the calculating process. It suggests us that the branching probability matrices $Q_{1}, Q_{2}, R_{1}, R_{2}$ and the unit inflow matrix $Q=\left(Q_{i j}\right)$, which we so called since $Q_{i j}$ means the influence of inflow of a single vehicle through ramp $i$ on flow over section $j$, should be estimated using both of the actual flows over sections and the actual in- and outflows through ramps. The constraint mentioned in the last paragraph may be settled after the error vector $c$ has been introduced so as to eliminate the difference between the traffic flow vectors $X$ and $Y$. Thus we may obtain every parameter involved in Eqs. (8), (13), (18) and (20). 


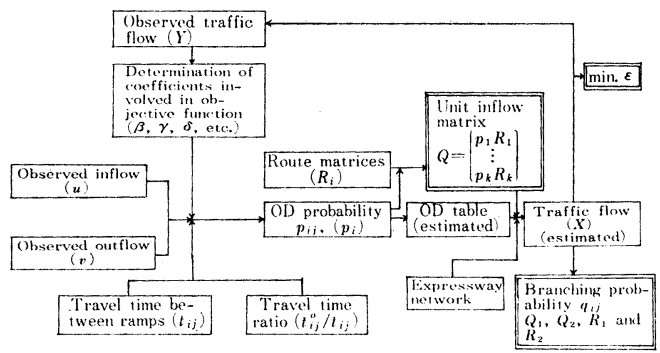

Fig. 5 Flow chart for estimating trip distributions between ramps on expressway

Traffic demand on expressway has usually just a few peaks at almost fixed hours within urban area. We should have only to control for the peak hour intervals in which traffic flow may be assumed approximately to be in steady state. Therefore our estimation should be sufficiently effective as well as practical. The estimation of trip distribution during a shorter interval is almost impossible without loss of estimation efficiency, since the travel time between ramps can not be disregarded and since Stiring Formula is not valid for lighter in-and outflows during such a short interval.

\section{CONTROL PRINCIPLE}

From the theoretical point of view, the more valid control is possible on the smaller scale expressway network, since the joint probability of occurrence of trips between ramps increases in its theoretical validity for such a smaller scale network on which travel time between ramps is presumably negligible. However, we have investigated in the control mainly for steady flow, which is acceptable on usual urban expressway network. For steady flow we have two kinds of control; Linear Programming control and Proportional control.

\section{(1) Linear Programming control}

The principle of Linear Programming control consists in realizing the maximum allowable inflow under the constraint that traffic flow should not be over the capacity over every section. Eqs. (8) and (18) are the constraints and Eq. (7) is the objective function. It has been made clear that the route matrix method brings us more secure control than the branching probability matrix method.

\section{(2) Proportional control}

In this control inflow through ramp $i$ is reduced by

$$
\Delta U_{i}=\Delta p_{j} U_{i}
$$

where,

$$
\begin{aligned}
& U_{i}=\text { inflow through ramp } i \\
& \Delta p_{j}=\Delta X_{j} / X_{j} \\
& \begin{aligned}
X_{j} & =\text { traffic flow over section } j \\
& =\sum_{i} U_{i} Q_{i j}
\end{aligned} \\
& \begin{aligned}
\Delta X_{j} & =\text { traffic flow over capacity over section } j \\
& =X_{j}-C_{j}>0
\end{aligned}
\end{aligned}
$$

Eq. (28) is led as follows. The contributon of in- flow $U_{i}$ through $\operatorname{ramp} i$ to traffic flow, $X_{j}=\sum_{i} U_{i} Q_{i j}$, over section $j$ is given by $U_{i} Q_{i j} / X_{j}$, since the traffic flow caused over section $j$ by the inflow $U_{i}$ is equal to $U_{i} Q_{i j}$ where $Q_{i j}$ is the $i j$-entry of the unit inflow matrix $Q$. Proportional control is carried out so that the contribution of inflow $\Delta U_{i}$, by which inflow $U_{i}$ through ramp $i$ is to be cut down, to the overflowed traffic flow $\Delta X_{j}$ over section $j$ should be equal to the contribution of $U_{i}$ to $X_{j}$. That is, we put

$$
\Delta U_{i} Q_{i j} / \Delta X_{j}=U_{i} Q_{i j} / X_{j}
$$

and so we get

$$
\begin{aligned}
\Delta U_{i} & =\left(\Delta X_{j} / X_{j}\right) U_{i} \\
& =\Delta p_{j} U_{i}
\end{aligned}
$$

Obviously, the sum $\sum_{i} \Delta U_{i} Q_{i j}$ is equal to $\Delta X_{j}$ by which traffic flow $X_{j}$ should be reduced.

Proportional control is carried out for those onramps alone which contribute traffic flows to the overflowed section. If there still exist any overflowed sections after the first Proportional control has been carried out, the same control is to be repeated until there exists no overflowed section.

\section{(3) Control by On-ramp Closing}

For unsteady flow we have to control by on-ramp closing, in which we close at first the on-ramp nearest by any overflowed section, and next the second nearest and so on if necessary. Estimation of inflow through each ramp is indispensable in this control.

Suppose that on-ramp closing is carried out every a fixed interval $\Delta t$, and that in respect to section $j$ the on-ramps are grouped into subsets of $A_{j_{1}}, A_{j_{2}}$, $\cdots, A_{j n}$ such that

i ) $A_{j_{1}}=$ subset of on-ramps $i^{\prime} s$ satisfying $\Delta t \geqq \tau_{i j}$

ii) $A_{j_{2}}=$ subset of on-ramps $i^{\prime} s$ satisfying $2 \Delta t \geqq$ $\tau_{i j}>\Delta t$

iii) $A_{j n}=$ subset of on-ramps $i^{\prime} s$ satisfying $n \Delta t \geqq$ $\tau_{i j}>(n-1) \Delta t$

where $\tau_{i j}$ is the travel time between on-ramp $i$ and specfied section $j$. From the economical viewpoint, the interval $\Delta t$ had better not been decided so that $A_{j_{1}}=0$ because, if $A_{j_{1}}=0$, we are to enforce on computer any operation in vain.

With those notations we have the constraint for section $j$ of the form of

$$
\begin{array}{r}
\sum_{i \in A_{j_{1}}} U_{i}\left(t-\tau_{i j}\right) Q_{i j}+\sum_{i \in A_{j_{2}}} U_{i}\left(t-\tau_{i j}\right) Q_{i j} \\
\quad+\cdots \leqq C_{j}-\varepsilon_{j} \cdots \cdots \cdots \cdots \cdots \cdots \cdots \cdots \cdots \cdots \cdots \cdots \cdots \cdots \cdots
\end{array}
$$

or alternatively

$$
\begin{array}{r}
\sum_{i \in A_{j_{1}}} U_{i}(t) Q_{i j}+\sum_{i \in A_{j_{2}}} U_{i}(t-\Delta t) \boldsymbol{Q}_{i j} \\
+\cdots \leqq C_{j}-\varepsilon_{j} \cdots \cdots \cdots \cdots \cdots \cdots \cdots
\end{array}
$$

If section $j$ is overflowed by traffic flow at time $t$, we close on-ramps $i^{\prime} s$ ( $i \in A_{j_{1}}$ ), that is, we put

$$
U_{i}=0 \text {, }\left(i \in A_{j_{1}}\right)
$$

We will have further closings at the same time if necessary. For instance, we close not only onramps $i^{\prime} s$ ( $i \in A_{j_{1}}$ ) but the $i^{\prime} s\left(i \in A_{j_{2}}\right.$ ), if 


$$
\begin{aligned}
\sum_{i \in A_{j_{2}}} U_{i}(t+\Delta t) Q_{i j}+\sum_{i \in A_{j_{3}}} U_{i}(t) Q_{i j} \\
\quad+\sum_{i \in A_{j_{4}}} U_{i}(t-\Delta t) Q_{i j}+\cdots>C_{j}-\varepsilon_{j} \cdots(30)
\end{aligned}
$$

in which $U_{i}(t+\Delta t)$, ( $i \epsilon A_{j_{2}}$ ), is to be forecasted. And in succession the $i^{\prime} s$, ( $i \in A_{j_{3}}$ ), , , will be closed, if required.

There may be any undesirable result that the full closing control for some requested on-ramps is likely to force those on-ramps alone to be blocked during some successive intervals. Such a result, however, will be avoided to some extent by imperfect closing principle instead of the full. Anyway it is a feeling that control of unsteady flow should imply some claptrap factors as compared with control of steady How. Unsteady flow has been perplexing us to settle criterion function.

\section{CONCLUSION}

There may be a variety of traffic control criterions on urban expressway network. There remins some ambiguity as to the practical measure of effectiveness of each control principle. Which is of practical validity will be made clear through any practical test. We are to make further investigation to find better control principle through simulation.

Another approach should be possible by taking account of economic operations. For instance, the congestion cost may be introduced into the measure of effectiveness of control system.

\section{ACKNOWLEDGMENT}

The authers are much indebted to Yasunori Iida, Reseach Assistant at Kyoto Univ., for his co-operation in investigation in paragraph 4 . The authers also wish to express their gratitude to the members of Research Committee for Traffic Control on Hanshin Expressway, set up by Express Highway Research Foundation of Japan, for their encouragement.

\section{References}

1) Tsuna Sasaki : "Theory of Traffic Assignment through Absorbing Markov Process", Trans. of Japan Society of Civil Engineers, No. 121, pp. 28-32, Sept. 1965.

2) ref. to 1).

3) Tsuna Sasaki : "Probabilistic Models for Trip Distributions", Traffic Engineering, Vol. 2 No. 6, pp. 1221, Nov. 1967.

(Received June, 20, 1968) 